PHYSICAL REVIEW D 92, 119904(E) (2015)

\title{
Erratum: Radiative improvement of the lattice nonrelativistic QCD action using the background field method with applications to quarkonium spectroscopy \\ [Phys. Rev. D 88, 014505 (2013)]
}

\author{
T. C. Hammant, A. G. Hart, G. M. von Hippel, R. R. Horgan, and C. J. Monahan
}

(Received 16 November 2015; published 14 December 2015)

Our paper contained several typographical errors and one mathematical error. The following corrections should be made: Equation (68) should read

$$
\begin{aligned}
& Z_{\mathrm{sym}-\sigma}^{\mathrm{QCD},(1)}=-\frac{1}{4 \pi}+\frac{1}{4 \pi} \log (\mu / M)+\frac{M}{6 \mu}, \\
& Z_{\mathrm{asym}-\sigma}^{\mathrm{QCD},(1)}=-\frac{1}{4 \pi} \log (\mu / M) .
\end{aligned}
$$

Equations (69)-(72) contain several typographical errors and should read

$$
\begin{aligned}
I_{\mathrm{sym}}^{\mathrm{sub}}(\mu) & =-\int \frac{d^{4} k}{(2 \pi)^{4}} \frac{4 \pi M^{2}\left(1+\mathbf{k}^{2} / 4 M^{2}\right)}{\left(k^{2}+\mu^{2}\right)^{2}\left(k_{0}^{2}+\mathbf{k}^{4} / 4 M^{2}\right)} \\
& =\tilde{Z}_{\mathrm{sym}}^{\mathrm{sub}}(\mu)-\frac{1}{\pi} \log (\mu a)-\frac{5 M}{16 \mu}+\frac{M^{2}}{2 \pi \mu^{2}}-\frac{M^{3}}{2 \mu^{3}} \\
I_{\text {asym }}^{\mathrm{sub}}(\mu) & =-\int \frac{d^{4} k}{(2 \pi)^{4}} \frac{4 \pi M^{2}}{\left(k^{2}+\mu^{2}\right)^{2}\left(i k_{0}+\mathbf{k}^{2} / 2 M\right)^{2}} \\
& =\tilde{Z}_{\text {asym }}^{\mathrm{sub}}(\mu)+\frac{15}{8 \pi} \log (\mu a)+\frac{3 M}{8 \mu}-\frac{M^{2}}{2 \pi \mu^{2}} \\
I_{\mathrm{sym}-\sigma}^{\mathrm{sub}}(\mu) & =-\int \frac{d^{4} k}{(2 \pi)^{4}} \frac{4 \pi}{\left(k^{2}+\mu^{2}\right)^{2}}\left(\frac{\mathbf{k}^{2} / 3}{k_{0}^{2}+\mathbf{k}^{4} / 4 M^{2}}+\frac{1}{2}\right) \\
= & \tilde{Z}_{\mathrm{sym}-\sigma}^{\mathrm{sub}}(\mu)-\frac{1}{4 \pi} \log (\mu a)-\frac{M}{6 \mu} \\
I_{\text {asym }-\sigma}^{\mathrm{sub}}(\mu)= & -\int \frac{d^{4} k}{(2 \pi)^{4}} \frac{4 \pi}{\left(k^{2}+\mu^{2}\right)^{2}}\left(\frac{\mathbf{k}^{2} / 3}{\left(i k_{0}+\mathbf{k}^{2} / 2 M\right)^{2}}-\frac{1}{2}\right) \\
= & \tilde{Z}_{\mathrm{asym}-\sigma}^{\mathrm{sub}}(\mu)+\frac{1}{4 \pi} \log (\mu a) .
\end{aligned}
$$

In Figs. $7-8$, the ordinate axis should be labeled $\left(-\tilde{Z}_{\mathrm{sym}-\sigma}^{\mathrm{sub}}\right)$ and $\left(-\tilde{Z}_{\mathrm{asym}-\sigma}^{\mathrm{sub}}\right)$, respectively, and in the corresponding captions the signs of $\tilde{Z}_{\mathrm{sym}-\sigma}^{\mathrm{sub}}(\mu)$ and $\tilde{Z}_{\mathrm{asym}-\sigma}^{\mathrm{sub}}(\mu)$ should be flipped accordingly. (Note that this error is purely presentational.)

As a consequence of the change in Eq. (68), the un-numbered equation at the beginning of Sec. VI.C should read

$$
\begin{aligned}
Z_{\mathrm{sym}-\sigma}^{(1)} & =-\tilde{Z}_{\mathrm{sym}-\sigma}^{\mathrm{NR},(1)}-\frac{1}{4 \pi}-\frac{1}{4 \pi} \log M a, \\
Z_{\mathrm{asym}-\sigma}^{(1)} & =-\tilde{Z}_{\mathrm{asym}-\sigma}^{\mathrm{NR},(1)}+\frac{1}{4 \pi} \log M a .
\end{aligned}
$$

Tables VI-VIII for the four-fermion coefficients $b_{1}$ and $b_{8}$ thus change as detailed below. This leads to a corresponding change in the one-loop corrected hyperfine splitting, and Tables IX-XI should be amended accordingly as detailed below.

In the conclusions, the sentence beginning "The resulting estimate for the hyperfine splitting" should therefore be changed to read "The resulting estimate for the hyperfine splitting of $68(3)(5)(3) \mathrm{MeV}$ is then in good agreement with the experimental value of 69.3(2.8) MeV." The general conclusions of our paper remain unaffected.

We are grateful to the authors of [3] for drawing our attention to the error in Eq. (68). 
TABLE VI. Renormalization parameters for spin-dependent four-fermion operators, $n=2$ full $v^{4}$ nonrelativistic QCD (NRQCD).

\begin{tabular}{lccc}
\hline \hline$M a$ & 1.95 & 2.8 & 4.0 \\
\hline$b_{1}$ & $0.0037(3)$ & $-0.0201(4)$ & $-0.0490(5)$ \\
$b_{8}$ & $0.0893(8)$ & $0.0183(15)$ & $-0.0832(26)$ \\
\hline \hline
\end{tabular}

TABLE VII. Renormalization parameters for spin-dependent four-fermion operators, $n=4$ full $v^{4}$ NRQCD.

\begin{tabular}{lccr}
\hline \hline$M a$ & 1.9 & 2.65 & 3.4 \\
\hline$b_{1}$ & $0.0075(1)$ & $-0.0148(6)$ & $-0.0341(2)$ \\
$b_{8}$ & $0.0997(5)$ & $0.0353(1)$ & $-0.0290(8)$ \\
\hline \hline
\end{tabular}

TABLE VIII. Renormalization parameters for spin-dependent four-fermion operators, $n=4$ full-spin $v^{6}$ NRQCD.

\begin{tabular}{lccr}
\hline \hline$M a$ & 1.9 & 2.65 & 3.4 \\
\hline$b_{1}$ & $-0.0223(2)$ & $-0.03599(2)$ & $-0.0504(2)$ \\
$b_{8}$ & $-0.0280(9)$ & $-0.0624(7)$ & $-0.1071(9)$ \\
\hline \hline
\end{tabular}

TABLE IX. Estimates of the corrections to the bottomonium hyperfine splitting results of [1] arising from the radiative improvement of the $n=2$ full $v^{4}$ NRQCD action. The errors given in the last column are statistical, $O\left(\alpha^{2}\right)$, and relativistic corrections, in that order.

\begin{tabular}{lccccccc}
\hline \hline$M a$ & $\beta$ & $\alpha_{V}$ & $c_{4}$ correction & Box correction & Total & Old hfs & New hfs \\
\hline 1.95 & 7.09 & 0.216 & $+31.4(3) \%$ & $-10.4(1) \%$ & $+21.0(3) \%$ & $56(2)$ & $68(3)(5)(6)$ \\
2.8 & 6.76 & 0.249 & $+39.8(3) \%$ & $+1.3(2) \%$ & $+41.1(4) \%$ & $50(2)$ & $71(3)(6)(5)$ \\
4.0 & 6.458 & 0.293 & $+49.3(3) \%$ & $+23.2(3) \%$ & $+72.5(4) \%$ & $41(2)$ & $71(3)(7)(4)$ \\
\hline \hline
\end{tabular}

TABLE $X$. The corrections to the bottomonium hyperfine splitting arising from the radiative improvement of the $n=4$ full $v^{4}$ NRQCD action, as found in [2]. Only the four-fermion contributions are post hoc estimates. The errors given in the last column are statistical, $O\left(\alpha^{2}\right)$, and relativistic corrections, in that order.

\begin{tabular}{|c|c|c|c|c|c|}
\hline \multirow[b]{2}{*}{$M a$} & \multirow[b]{2}{*}{$\alpha_{V}$} & \multicolumn{2}{|c|}{ Hfs (MeV) } & \multirow{2}{*}{$\begin{array}{l}\text { Correction } \\
\text { four-fermion }\end{array}$} & \multirow{2}{*}{$\begin{array}{c}\text { Hfs }(\mathrm{MeV}) \\
\text { corrected }\end{array}$} \\
\hline & & $c_{4}=1$ & Improved $c_{4}$ & & \\
\hline 1.9 & 0.225 & $56.1(1)$ & $72.1(1)$ & $-12.6(1) \%$ & $65.0(1)(2.8)(5.6)$ \\
\hline 2.65 & 0.253 & $50.5(1)$ & $69.8(1)$ & $-1.8(1) \%$ & $68.9(1)(3.2)(5.0)$ \\
\hline 3.4 & 0.275 & $45.6(1)$ & $65.6(1)$ & $+11.0(1) \%$ & $70.6(1)(3.4)(4.6)$ \\
\hline
\end{tabular}

TABLE XI. Corrections to the bottomonium hyperfine splitting arising from the radiative improvement of the $n=4$ full NRQCD action including spin-dependent terms at order $v^{6}$.

\begin{tabular}{lccc}
\hline \hline$M a$ & $\alpha_{V}$ & $c_{4}$ correction & Box correction \\
\hline 1.9 & 0.225 & $+37(4) \%$ & $+7.7(1) \%$ \\
2.65 & 0.253 & $+40(4) \%$ & $+15.4(1) \%$ \\
3.4 & 0.275 & $+26(4) \%$ & $+25.9(1) \%$ \\
\hline \hline
\end{tabular}

[1] A. Gray, I. Allison, C. T. H. Davies, E. Gulez, G. P. Lepage, J. Shigemitsu, and M. Wingate, Phys. Rev. D 72, 094507 (2005).

[2] R. Dowdall et al. (HPQCD Collaboration), Phys. Rev. D 85, 054509 (2012).

[3] M. Baker, A. Penin, D. Seidel, and N. Zerf, Phys. Rev. D 92, 054502 (2015). 